\title{
Determination of Aqueous Formic and Acetic Acids by Purge-and- Trap Analysis with a Needle-Type Extraction Device and Gas Chromatography Barrier Discharge Ionization Detector
}

\author{
Ikuo Ueta, ${ }^{* \dagger}$ Yohei NaKamura, ${ }^{*}$ Susumu KawaKubo, ${ }^{*}$ and Yoshihiro Saito** \\ *Department of Applied Chemistry, University of Yamanashi, 4-3-11 Takeda, Kofu 400-8511, Japan \\ **Department of Environmental and Life Sciences, Toyohashi University of Technology, Toyohashi 441-8580, \\ Japan
}

\begin{abstract}
A purge-and-trap method using a needle-type extraction device and a gas chromatography-barrier discharge ionization detector for the analysis of formic (FA) and acetic acids (AA) in aqueous samples is presented. An activated carbon-based adsorbent, Carboxen 1000, was employed as the extraction medium for the needle-type extraction device. The sampling time was $5 \mathrm{~min}$ for collecting headspace gas in a glass vial, including $10 \mathrm{~mL}$ of an aqueous sample. The detection limits for FA and AA with a headspace sampling volume of $100 \mathrm{~mL}$ were 3.3 and $2.0 \mathrm{mg} \mathrm{L}^{-1}$, respectively. Since the proposed method was based on purge-and-trap collection, it was suitable for the determination of aqueous FA and AA in complex matrices with simple and rapid sample preparation steps. The proposed method was applied to the determination of FA and AA in fruit juice samples and FA generated by the electrochemical reduction of carbon dioxide.
\end{abstract}

Keywords Needle extraction, purge-and-trap, formic acid, acetic acid

(Received September 5, 2017; Accepted September 25, 2017; Published February 10, 2018)

\section{Introduction}

Formic acid (FA) and acetic acid (AA) are low-molecularweight carboxylic acids, which are present in foods, beverages, plants, and environmental samples. ${ }^{1,2}$ The determinations of FA and AA are important for quality control in food and beverage manufactures. These acids are also important as intermediates for several compounds. They have several undesirable effects on the human body, such as irritation of the eyes, nose, throat, and skin, at relatively high concentrations of liquid or vapor. Because FA is synthesized by the reduction of carbon dioxide $\left(\mathrm{CO}_{2}\right)$, it is also important in terms of energy storage. ${ }^{3}$

$\mathrm{FA}$ and $\mathrm{AA}$ in aqueous samples are typically determined by ion chromatography (IC) with an electrical conductivity detector. ${ }^{4}$ American Society for Testing and Materials (ASTM) requires detection limits of $\mathrm{FA}$ and $\mathrm{AA}$ in highly pure water at few $\mu \mathrm{g} \mathrm{L}^{-1}$ with a sample preconcentration process. ${ }^{5}$ The IC method provides sensitive determinations of both FA and AA; however, co-elution of these compounds with fluorides should be problem. In addition, adequate sample preparation processes are required for the analysis of complex matrices before injection to remove interferences. Gas chromatography (GC) is also used for the determination of FA and AA. ${ }^{6}$ In GC analysis, mass spectrometry is often employed as a detector, ${ }^{7-9}$ while other conventional GC detectors, such as flame ionization detector (FID) or thermal conductivity detector, have poor sensitivity to the analytes. FID is not suitable for the

† To whom correspondence should be addressed.

E-mail: iueta@yamanashi.ac.jp determination of FA because of its lower response for FA. The barrier discharge ionization detector (BID) is a recently developed GC detector. This is a universal detector, which enables the sensitive detection of all volatile compounds, except for helium and neon. ${ }^{10,11}$ The application of GC-BID to the determination of VOCs are still very limited, and especially, reports for the determination of VOCs with a sample preparation step would be beneficial and meaningful for researchers.

For the determination of trace volatile organic compounds (VOCs) in aqueous samples by GC, adequate sample preparation is required. ${ }^{12}$ Solid-phase microextraction (SPME) is one of the most popular methods for the sample preparation of VOCs, ${ }_{13}^{13,14}$ but this method is suitable for low-volatility organic compounds because the analytes were extracted on the extraction phase based on the equilibrium between the sample matrix and the extraction phase. Solid-phase extraction (SPE) is also used for the extraction of low-volatility and relatively hydrophobic organic compounds. ${ }^{15}$ The needle-type extraction device is a miniaturized extraction device designed for GC analysis, ${ }^{16-19}$ which also offers a solvent-free technique for the determination of VOCs. The analytes are extracted onto a particulate sorbent packed into the stainless-steel needle by dynamic sampling of gaseous samples, and then the extracted analytes are thermally desorbed in the heated conventional GC injection port. ${ }^{20,21}$ The needle-type extraction device has been utilized for the determination of several VOCs in gaseous samples, such as the analysis of VOCs related to sick building syndrome, ${ }^{22}$ herbal medicines, ${ }^{23}$ and human breath. ${ }^{24,25}$ The device was also applied to the determination of VOCs in aqueous samples based on the purge-and-trap (PT) method. ${ }^{26-29}$ PT method is suitable for the sample preparation of complex aqueous samples including non- 
volatile compounds, such as sugars and salts. For PT analysis with the needle device, the extraction needle was inserted into the headspace of a glass vial that included an aqueous sample, and the headspace gas was dynamically collected through the extraction needle. During the dynamic sampling, a purge gas (nitrogen) is bubbled through the aqueous sample due to decompression in the glass vial. Therefore, PT analysis with a needle-type extraction device does not require special or expensive instruments, because it allows for simple and rapid determinations of trace VOCs in aqueous samples. In addition, PT extraction with a needle-type extraction device achieved a lower detection limit than SPME for the determination of FA and $\mathrm{AA}$ in aqueous samples. ${ }^{30}$

Previously, we reported the extraction of FA and AA from gaseous samples with a needle-type extraction device, followed by GC-BID analysis. ${ }^{31}$ In that study, gaseous FA and AA were sensitively determined with a simple and rapid sample preparation step and the method was successfully applied for analyzing in-door air samples. In this study, the determination of FA and AA in aqueous samples by PT extraction using a needle-type extraction device with GC-BID is reported. Several experimental conditions are quantitatively evaluated in this paper, and then quantitative performances and the applicability of the proposed method are discussed.

\section{Experimental}

\section{Chemicals}

FA $(99.5 \%)$, AA (>99.9\%), sulfuric acid $\left(\mathrm{H}_{2} \mathrm{SO}_{4}\right)$, and sodium hydroxide $(\mathrm{NaOH})$ were purchased from Wako Pure Chemical Industries, Ltd. (Osaka, Japan). Sodium chloride $(\mathrm{NaCl})$ was obtained from Kanto Chemical Co., Inc. (Tokyo, Japan).

\section{Needle-type extraction device}

A stainless-steel needle $(85 \mathrm{~mm} \times 0.5 \mathrm{~mm}$ i.d., $0.7 \mathrm{~mm}$ o.d. $)$ with a tip hole was used in this study. An activated carbonbased sorbent, Carboxen 1000 (Sigma-Aldrich Japan, Tokyo, Japan), was packed into the needle as the extraction medium. In our previous study, Shincarbon ST was used as the adsorbent for the extraction of FA and AA in gaseous samples. ${ }^{31}$ In this study, Carboxen 1000 showed a lower adsorption of moisture than other activated carbon-based sorbents, owing to its relatively hydrophobic surface. Because a large amount of moisture extracted on the adsorbent leads to a decrease in the peak intensity and also the repeatability, a hydrophobic adsorbent is preferable in PT analysis. The particle size was 60/80 mesh $(180-250 \mu \mathrm{m})$, and the packing length of the sorbent was fixed at $25 \mathrm{~mm}$. A small amount of heat-resistant polymeric filaments of Zylon (Toyobo Co., Ltd., Osaka, Japan) was also packed at each end of the packed section to fix the sorbent.

\section{Sampling and desorption methods}

A standard aqueous $10 \mathrm{~mL}$ sample was prepared in a $20-\mathrm{mL}$ glass vial. To insert the extraction needle and a stainless-steel needle that supplied the purge gas, two silicon septa were inserted in the plastic cap of the vial. Then, $\mathrm{NaCl}$ and $\mathrm{H}_{2} \mathrm{SO}_{4}$ were added to the solution, which was sonicated for $1 \mathrm{~min}$ to dissolve $\mathrm{NaCl}$. The vial was then immersed in a water bath kept at a constant temperature for $5 \mathrm{~min}$ while the solution was being stirred with a polytetrafluoroethylene-coated magnetic stirring bar at $350 \mathrm{rpm}$. The water bath was heated with a hot-plate stirrer. The amounts of $\mathrm{NaCl}$ and $\mathrm{H}_{2} \mathrm{SO}_{4}$ and the temperature of the water bath (PT temperature) were optimized in the following study. The extraction needle, which was attached to a vacuum

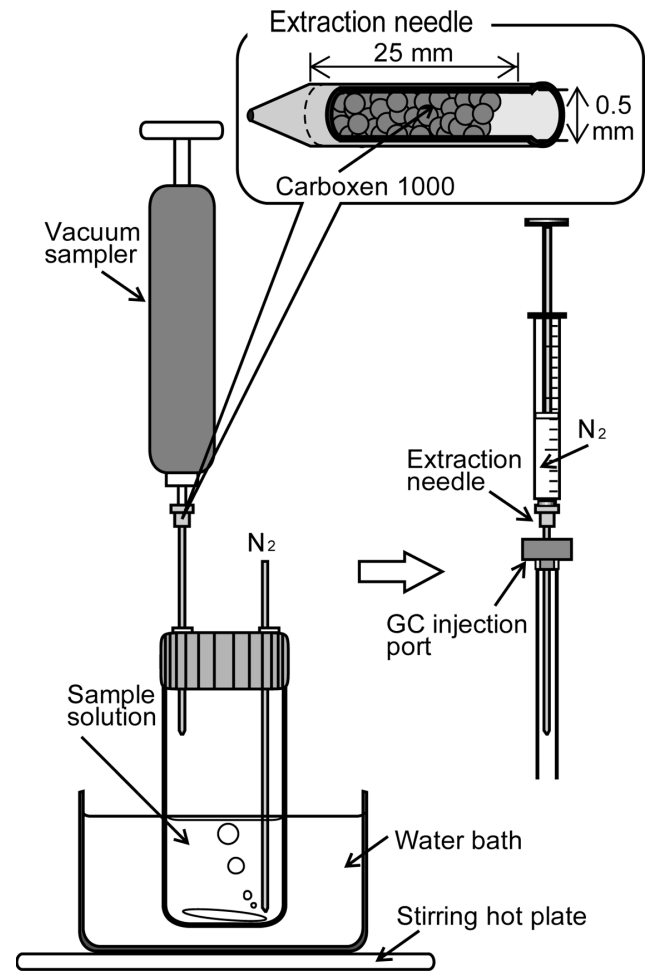

Fig. 1 Illustration of PT extraction with a needle-type extraction device and GC-BID analysis.

sampling device (Komyo Rikagaku Kogyo, Tokyo, Japan), was inserted into the vial through a silicon septum. A blank stainless-steel needle was inserted into the aqueous sample through another septum, and this needle was connected to a gas sampling bag (Smart Bag 2F, GL Sciences, Tokyo, Japan) filled with $\mathrm{N}_{2}$ gas. During the active sampling of the head space gas, the purge gas $\left(\mathrm{N}_{2}\right)$ was continuously supplied into the aqueous solution. The sampling time for the extraction of $100 \mathrm{~mL}$ of headspace gas with the extraction needle was approximately $5 \mathrm{~min}$. After extraction, $100 \mathrm{~mL}$ of $\mathrm{N}_{2}$ gas was collected through the extraction needle using the vacuum sampling device to remove excess water from the extraction needle. An illustration of the PT method used in this study is given in Fig. 1.

The extraction needle was then attached to a gas tight syringe (1.0 mL volume), and $0.5 \mathrm{~mL}$ of desorption gas $\left(\mathrm{N}_{2}\right)$ was collected via the extraction needle. The needle was inserted into the heated GC injection port, and then the analytes were thermally desorbed from the extraction needle before being introduced into the GC column by the desorption gas over $5 \mathrm{~s}$.

\section{$G C$ measurement}

A Shimadzu GC-2010 Plus capillary gas chromatograph with a BID (Tracera, Shimadzu Corporation, Kyoto, Japan) was used for all of the GC measurements. An HP-INNOWAX capillary column, $25 \mathrm{~m} \times 0.20 \mathrm{~mm}$ i.d., with polyethylene glycol phase of $0.4 \mu \mathrm{m}$ film thickness (Agilent Technologies, Santa Clara, CA, USA) were used for the separation of the analytes. Helium $(>99.999 \%)$ was used as the carrier gas at a column head pressure of $150 \mathrm{kPa}$. All of the injections were performed using a split mode with a ratio of 10:1, and the injector and detector temperatures were set at 250 and $270^{\circ} \mathrm{C}$, respectively. The column temperature was initially maintained at $100^{\circ} \mathrm{C}$ for $2 \mathrm{~min}$, and then increased to $200^{\circ} \mathrm{C}$ at $25^{\circ} \mathrm{C} \mathrm{min}{ }^{-1}$. The relative standard 

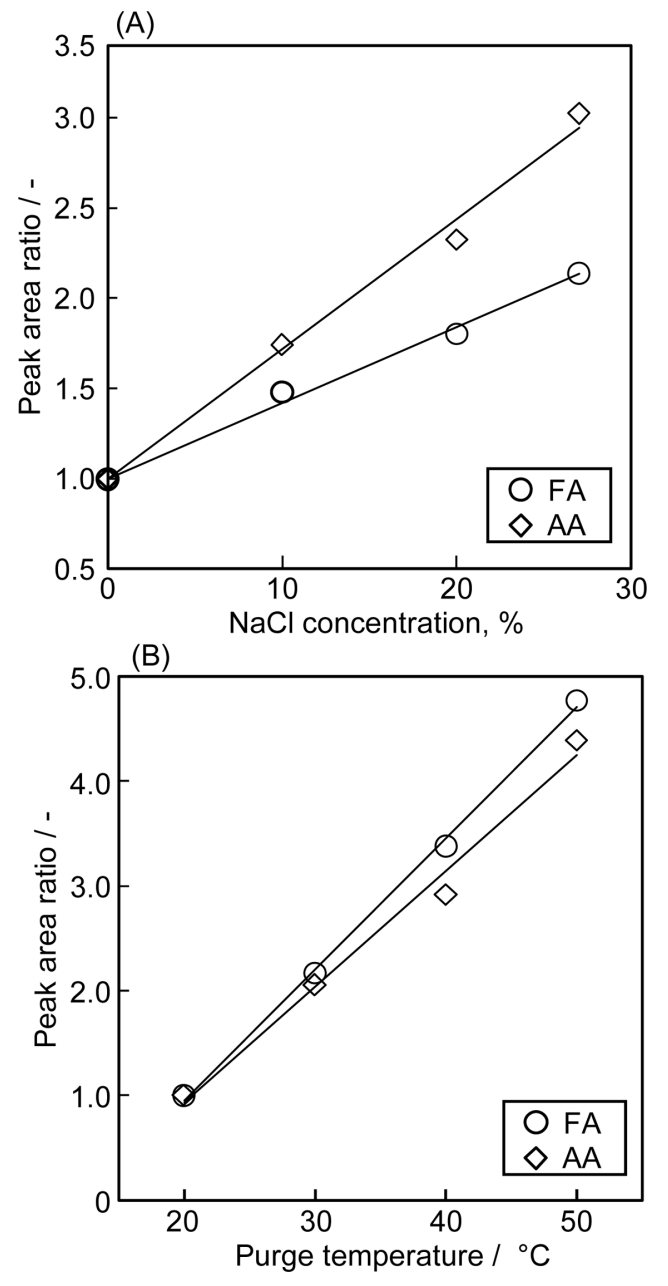

Fig. 2 Optimization of the PT method. (A) Concentration of $\mathrm{NaCl}$ and (B) purge temperature.

deviations (RSDs) for the peak area of standard gaseous sample for intraday and day-to-day measurements were less than 11 and $13 \%$, respectively.

\section{Real samples}

Fruit juices (apple, lime, grape, and orange) that were not from concentrate were purchased at a local market in Kofu, Japan. The juice samples were diluted with pure water five times to avoid bubble formation during the PT extraction. Aqueous solutions obtained by electrochemical reduction of $\mathrm{CO}_{2}$ with metal $(\mathrm{Pd})$ catalysts were also analyzed to determine the concentration of FA. Before the analysis, the solution was diluted ten times with pure water.

\section{Results and Discussion}

\section{Development of the PT method}

The purge efficiency of VOCs is typically increased by the addition of a salt into the sample solution in the PT method owing to a salting-out effect. The effects of the addition of $\mathrm{NaCl}$ on the PT analysis of FA and AA are shown in Fig. 2A. Both peak areas were calculated based on the ratio to the peak area obtained with a $\mathrm{NaCl}$ concentration of $0 \%$. The peak areas for both FA and AA increased with the $\mathrm{NaCl}$ concentration. The result was consistent with our previous work, in which the
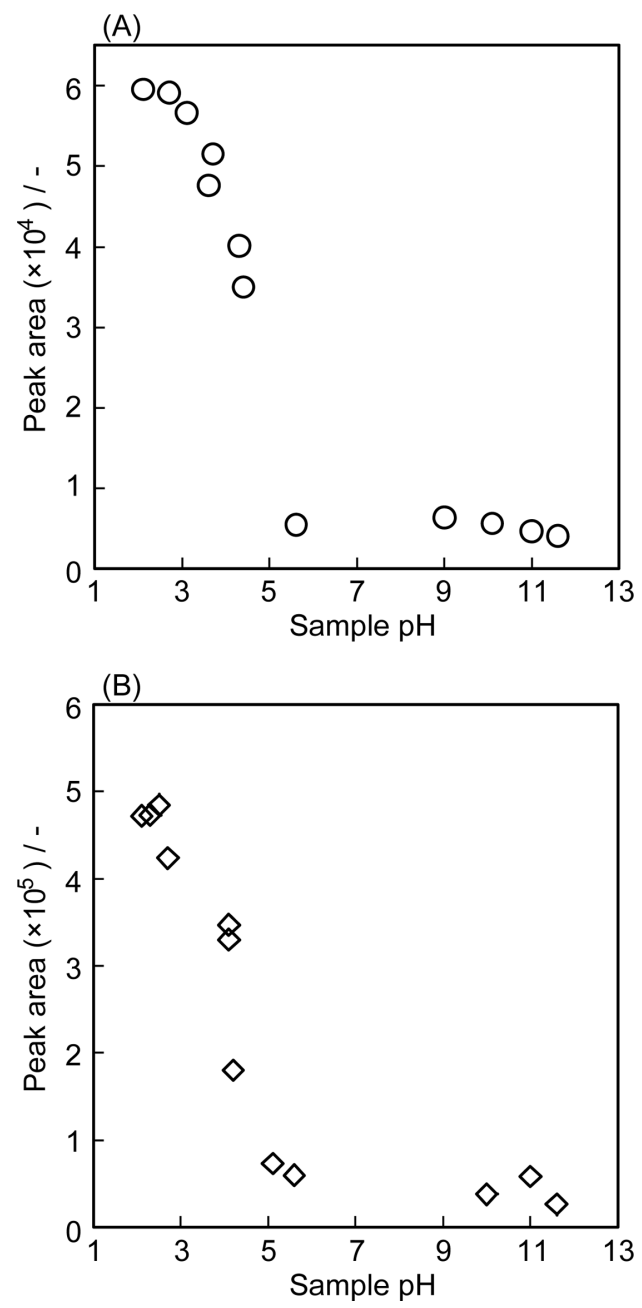

Fig. 3 Effect of the sample pH on the PT analysis of (A) FA and (B) AA.

peak areas of hydrophilic VOCs increased with the addition of $\mathrm{NaCl}$ in the PT extraction. ${ }^{29}$ Based on this result, the $\mathrm{NaCl}$ concentration was fixed at $26 \%$ (saturated concentration).

The effect of the purge temperature (water bath temperature) was investigated in the range from 20 to $50^{\circ} \mathrm{C}$. As shown in Fig. 2B, the peak areas increased with the purge temperature, although sampling of the headspace gas was quite difficult at a purge temperature of $50^{\circ} \mathrm{C}$, because of the condensation of vapor in the extraction needle. Therefore, a purge temperature of $40^{\circ} \mathrm{C}$ was chosen as the optimum temperature in this study.

Because FA $\left(\mathrm{p} K_{\mathrm{a}} 3.8\right)$ and AA $\left(\mathrm{p} K_{\mathrm{a}} 4.8\right)$ are weak acids, the $\mathrm{pH}$ of the sample solution is important with respect the purge efficiency of these analytes. The $\mathrm{pH}$ was adjusted by the addition of $0.1 \mathrm{~mol} \mathrm{~L}^{-1} \mathrm{H}_{2} \mathrm{SO}_{4}$ or $0.1 \mathrm{~mol} \mathrm{~L}^{-1} \mathrm{NaOH}$ solution. Figure 3 shows the effect of the $\mathrm{pH}$ on the analyte peak areas. The amounts of both FA and AA increased at $\mathrm{pH}$ values below the $\mathrm{p} K_{\mathrm{a}}$ values. Based on this result, the $\mathrm{pH}$ of the sample solution was adjusted to below 2 by addition of $\mathrm{H}_{2} \mathrm{SO}_{4}$ solution (0.1 $\left.\mathrm{mol} \mathrm{L}^{-1}\right)$.

The sampling volume of the dry purge gas used after the PT extraction was investigated with $0,50,100$, and $200 \mathrm{~mL}$, where the sampling speed was set at $20 \mathrm{~mL} \mathrm{~min}{ }^{-1}$. At a dry purge gas volume of $0 \mathrm{~mL}$ (without dry purge), a base-line increase was obtained due to the appearance of a large water peak, which effects the sensitivities of the analytes. The introduction of a 
Table 1 LOD and LOQ of FA and AA in this study

\begin{tabular}{lcc}
\hline & FA & AA \\
\hline $\mathrm{LOD} / \mathrm{mg} \mathrm{L}^{-1}$ & 3.3 & 2.0 \\
$\mathrm{LOQ} / \mathrm{mg} \mathrm{L}^{-1}$ & 10 & 6.0 \\
\hline
\end{tabular}

Table 2 Quantitative results for FA and AA in fruit juices

\begin{tabular}{lcc}
\hline & \multicolumn{2}{c}{ Concentration $/ \mathrm{mg} \mathrm{L}^{-1}$} \\
\cline { 2 - 3 } & FA & AA \\
\hline Apple & N.D. & N.D. \\
Lime & N.D. & N.D. \\
Orange & 201 & 30.8 \\
Grape & 45.6 & 25.4 \\
\hline
\end{tabular}

N.D.: Not detected.

dry purge process, significantly decreased the water peak, and the effects on the analysis of the analytes also disappeared. Taking into account the water peak and dry purge time, a volume of $100 \mathrm{~mL}$ of nitrogen gas was chosen for the dry purge process.

The relationships between the sampling volume of the headspace gas and the analyte peak areas were studied in the range from 100 to $600 \mathrm{~mL}$ (sampling time from 5 to $30 \mathrm{~min}$ ). The peak areas of both FA and AA linearly increased up to a sampling volume of $600 \mathrm{~mL}$ with correlation coefficients of more than 0.99 .

\section{Evaluation and application of the method}

The sensitivity and repeatability of the optimized PT method were investigated. The limit of quantification (LOQ) and the limit of detection (LOD) for a headspace gas sampling volume of $100 \mathrm{~mL}$ are given in Table 1. The LOQ was determined at a signal-to-noise ratio of 10 , and the LOD was determined at a ratio of 3. The sensitivities for both FA and AA obtained in this study are less than for typical ion chromatographic analysis, although the method enables the determination of FA and AA at a level of a few $\mathrm{mg} \mathrm{L}^{-1}$ from complex matrices, such as those including inorganic salts or non-VOCs. The upper limit of quantification was determined to be more than $2000 \mathrm{mg} \mathrm{L}^{-1}$. The repeatability of the method was confirmed by analyzing a standard solution ( $50 \mathrm{mg} \mathrm{L}^{-1}$ each) five times. The RSDs of the peak areas of FA and AA were 10.2 and $8.9 \%$, respectively. The RSDs of needle-to-needle comparison using five different extraction needles were less than $11.0 \%$.

The proposed method was applied to the analysis of real samples. $\mathrm{NaCl}$ and $\mathrm{H}_{2} \mathrm{SO}_{4}$ solution were also added to real samples to adjust the optimum $\mathrm{NaCl}$ concentration and the $\mathrm{pH}$, respectively. First, the FA and AA levels in fruit juice samples (apple, lime, orange, and grape) were measured. The quantitative results for the analytes in fruit juices are given in Table 2. As shown in the table, FA and AA were not detected in apple and lime juices, while small amounts of FA and AA were detected in orange and grape juices. A typical chromatogram for the determination of FA and AA in the orange juice ( 5 times diluted) is shown in Fig. 4. In this study, a relatively small water peak was appeared about 2.2 min and co-eluted with a large peak. The recoveries of the analytes from the juice samples were also investigated. The recovery was calculated by the addition of a standard solution with a concentration of $50 \mathrm{mg} \mathrm{L}^{-1}$ into the diluted grape and orange juices. The recoveries of FA and AA

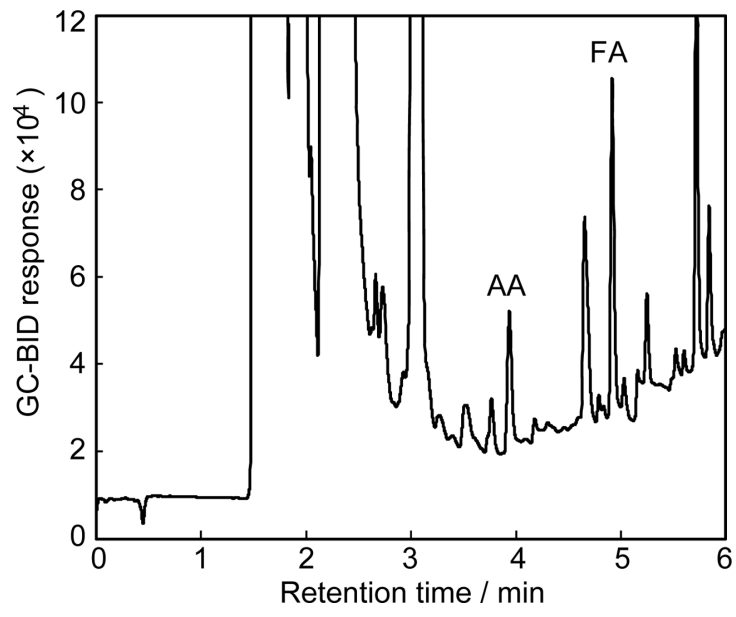

Fig. 4 Typical chromatogram for the determination of FA and AA in a diluted orange juice sample.

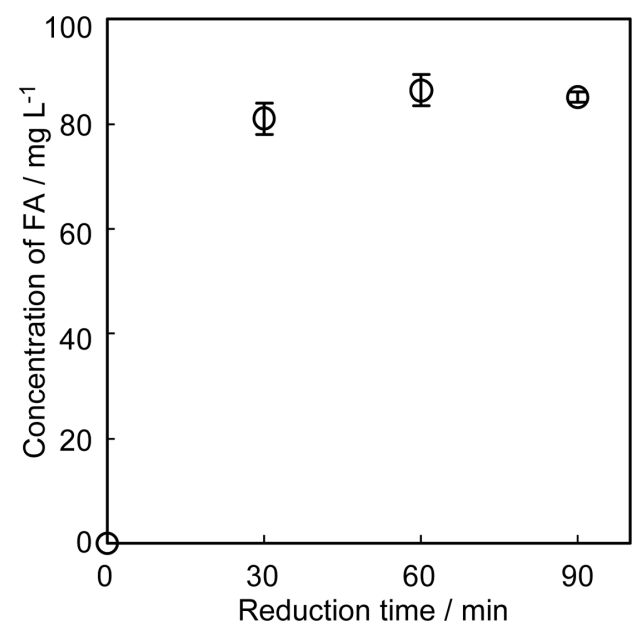

Fig. 5 Concentration of FA during the electrochemical reduction of $\mathrm{CO}_{2}$.

from the diluted juices were 93.4 and $98.7 \%$, respectively $(n=5)$. The RSDs of the peak area of FA and AA in fruit juices were less than $13 \%$.

The concentration of FA was determined for an aqueous solution that was obtained after the electrochemical reduction of $\mathrm{CO}_{2}$ using a metal catalyst. FA can be generated by the electrochemical reduction of $\mathrm{CO}_{2} \cdot{ }^{32}$ The amount of FA in the solution is an indicator of the catalytic activity and therefore, quantification of FA during the electrochemical reduction of $\mathrm{CO}_{2}$ is meaningful. ${ }^{33}$ Because the aqueous solution includes sodium hydrogen carbonate, direct injection of the solution into the GC should be avoided, and sample preparation using the PT method could be suitable. Figure 5 indicates the concentration of FA in an aqueous solution produced by the electrochemical reduction of $\mathrm{CO}_{2}$ with a $\mathrm{Pd}$ catalyst. For the determination of FA, $1 \mathrm{~mL}$ of the solution was sampled every $30 \mathrm{~min}$, and the collected sample was diluted with $4 \mathrm{~mL}$ of pure water. The concentration of FA increased with the electrochemical reduction of $\mathrm{CO}_{2}$ which almost reached a plateau at a reduction time of 30 min. The concentration of FA was less than LOQ at a reduction time of $15 \mathrm{~min}$. Based on these results, the proposed method could also be suitable for evaluating of the catalytic activity and reduction efficiency of $\mathrm{CO}_{2}$. 


\section{Conclusions}

This study demonstrated the PT extraction of FA and AA from aqueous solution with a needle-type extraction device followed by GC-BID analysis. The proposed method enables the determination of FA and AA in complex matrices at levels of a few $\mathrm{mg} \mathrm{L}^{-1}$ with simple sample preparation steps. The method could be applied to the determination of FA and AA in environmental and food samples as well as for the evaluation of catalytic activity. PT extraction with the needle-type extraction device coupled with GC-BID could be further applied to the determination of other low-molecular-weight organic compounds in aqueous samples.

\section{Acknowledgements}

Part of this study was financially supported by Shimadzu Science Foundation and Grant-in Aid for Scientific Research from the Japan Society for the Promotion of Science (JSPS) KAKENHI (Grant Number 15K17875 and 15K05537). The authors also acknowledge to Prof. Toshihiro Takashima and Yasutada Suzuki (University of Yamanashi) for their technical support.

\section{References}

1. H. Zom and P. Czermak, "Biotechnology of Food and Feed Additives", 2014, Springer, Heidelberg.

2. A. A. Randall, L. D. Benefield, W. E. Hill, J. P. Nicol, G. K. Boman, and J. Shu-Ren, Water Sci. Technol., 1997, 35, 153.

3. W. H. Wang, Y. Himeda, J. T. Muckerman, G. F. Manbeck, and E. Fujita, Chem. Rev., 2015, 115, 12936.

4. H. Fan, M. Ding, K. Tanaka, and W. Hu, Anal. Sci., 2005, 21,121

5. ASTM D5542-16, "Standard Test Methods for Trace Anions in High Purity Water by Ion Chromatography", 2016, ASTM International, West Conshohocken.

6. NIOSH Manual of Analytical Methods, Method 1603 Issue 2, 1994, National Institute for Occupational Safety and Health (NIOSH), Washington, D.C.

7. L. Alonso and M. J. Fraga, J. Chromatogr. Sci., 2001, 39, 297.

8. M. Ryhl-Svendsen and J. Glastrup, Atmos. Environ., 2002 , 36, 3909 .

9. J. Lu, Z. P. Wu, W. J. Chen, Y. P. Xian, X. D. Guo, J. X. Lv, and H. Li, Anal. Sci., 2016, 32, 407.

10. L. A. Frink, C. A. Weatherly, and D. W. Armstrong, J. Pharm. Biomed. Anal., 2014, 94, 111.

11. F. A. Franchina, M. Maimone, D. Sciarrone, G. Purcaro, P. Q. Tranchida, and L. Mondello, J. Chromatogr. A, 2015, 1402, 102.

12. A. C. Soria, M. J. García-Sarrió, and M. L. Sanz, Trends Anal. Chem., 2015, 71, 85.

13. G. Vas and K. Vékey, J. Mass Spectrom., 2004, 39, 233.

14. K. Demeestere, J. Dewulf, B. D. Witte, and H. V. Langenhove, J. Chromatogr. A, 2007, 1153, 130.

15. J. Xi, J. Zhang, and H. Zhao, Anal. Sci., 2017, 33, 999.

16. H. L. Lord, W. Zhan, and J. Pawliszyn, Anal. Chim. Acta, 2010, 677, 3 .

17. I. Ueta, Chromatography, 2013, 34, 23.

18. I. Ueta and Y. Saito, Anal. Sci., 2014, 30, 105.

19. K. Kędziora and W. Wasiak, J. Chromatogr. A, 2017, 1505, 1.

20. Y. Saito, I. Ueta, K. Kotera, M. Ogawa, H. Wada, and K. Jinno, J. Chromatogr. A, 2006, 1106, 190.

21. P. Trefz, S. Kischkel, D. Hein, E. S. James, J. K. Schubert, and W. Miekisch, J. Chromatogr. A, 2012, 1219, 29.

22. I. Ueta, A. Mizuguchi, K. Fujimura, S. Kawakubo, and Y. Saito, Anal. Chim. Acta, 2012, 746, 77.

23. Y. Qin, Y. Pang, and Z. Cheng, Phytochem. Anal., 2016, 27, 364.

24. M. Mieth, J. K. Schubert, T. Gröger, B. Sabel, S. Kischkel, P. Fuchs, D. Hein, R. Zimmermann, and W. Miekisch, Anal. Chem., 2010, 82, 2541.

25. I. Ueta, A. Mizuguchi, M. Okamoto, H. Sakamaki, M Hosoe, M. Ishiguro, and Y. Saito, Clin. Chim. Acta, 2014, $430,156$.

26. I. Ueta, T. Mitsumori, S. Kawakubo, and Y. Saito, Anal. Sci., 2014, 30, 979.

27. I. Ueta, T. Mitsumori, Y. Suzuki, S. Kawakubo, and Y. Saito, J. Chromatogr. A, 2015, 1397, 27.

28. I. Ueta, T. Mitsumori, Y. Suzuki, S. Kawakubo, and Y. Saito, Chromatography, 2015, 36, 99.

29. I. Ueta, N. A. Razak, A. Mizuguchi, S. Kawakubo, Y. Saito, and K. Jinno, J. Chromatogr. A, 2013, 1317, 211.

30. X. Lee, D. Huang, D. Lou, and J. Pawliszyn, J. Sep. Sci., 2012, 35, 1675.

31. I. Ueta, Y. Nakamura, K. Fujimura, S. Kawakubo, and Y. Saito, Chromatographia, 2017, 80, 151.

32. J. F. Hull, Y. Himeda, W-H. Wang, B. Hashiguchi, R. Periana, D. J. Szalda, J. T. Muckerman, and E. Fujita, Nat. Chem., 2012, 4, 383.

33. T. Takashima, T. Suzuki, and H. Irie, J. Photon. Energy, 2016, 7, 012005. 